VIII: Miscellaneous

\title{
29. The individualised Pharmacologic approach of Enhanced Recovery After Surgery pathways: Analgesics and local anaesthetics
}

Georgios S. Papageorgiou ${ }^{1, *}$, Vangelis G. Manolopoulos ${ }^{2}$

${ }^{1}$ Clinical Pharmacology and Therapeutics, Democritus University of Thrace, Alexandroupolis, Greece; ${ }^{2}$ the Laboratory of Pharmacology, Democritus University of Thrace, Alexandroupolis, Greece. * dr.georgios.papageorgiou@gmail.com

Background: Enhanced Recovery After Surgery (ERAS) is multidisciplinary, evidence-based approach in the perioperative care of surgical patients that aims to reduce postoperative complications, length of hospital stay, readmissions and healthcare costs by implementing protocols throughout patient's perioperative journey. (1) The purpose of this review is to highlight the pharmacologic perspective of the most common drugs utilised in ERAS protocols, present current evidence regarding optimal and individualised use of them, discuss ways how clinicians can maximise the benefits of their patients and outline future advancements in the field.

Methods: A wide literature search was performed to identify high quality evidence on the pharmacology of common analgesics and local anaesthetics used in ERAS protocols. PubMed, Embase and Scopus databases were searched using various combinations of terms related to perioperative analgesia, personalised/precision/individualised medicine, local anaesthetics and Enhanced Recovery after Surgery protocols, up to January 2021.

Results: Literature search revealed known and less known pharmacological properties of common analgesics and local anaesthetics, unveiled pharmacodynamic, pharmacokinetic and pharmacogenomic properties of drugs that can improve efficiency and reduce adverse-effects with a more individualised patient approach and suggested evidence-based ways of serving the purposes of ERAS protocols.

Conclusions: Clinical Pharmacology plays an essential role in improving patient care within ERAS pathways by implementing basic and advanced pharmacologic properties and pharmacogenomic data towards a more patient-centered approach.

\section{Reference}

[1] ERAS Society. Enhanced Recovery After Surgery Society. 2020. Available at: www.erassociety.org (Accessed: 20 November 2020). 